\title{
Clinicopathological and prognostic significance of metastasis- associated in colon cancer-1 (MACC1) overexpression in colorectal cancer: a meta-analysis
}

\author{
Yang Zhao ${ }^{1, *}$, Cong Dai ${ }^{1,}{ }^{*}$, Meng Wang ${ }^{1}$, Huafeng Kang ${ }^{1}$, Shuai Lin ${ }^{1}$, Pengtao Yang ${ }^{1}$, \\ Xinghan Liu ${ }^{1}$, Kang Liu ${ }^{1}$, Peng $X u^{1}{ }^{1}$, Yi Zheng ${ }^{1}$, Shanli Li ${ }^{1}$ and Zhijun Dai ${ }^{1}$ \\ ${ }^{1}$ Department of Oncology, Second Affiliated Hospital of Xi'an Jiaotong University, Xi'an, China \\ * These authors have contributed equally to this work and share joint first authorship
}

Correspondence to: Zhijun Dai, email: dzj0911@126.com

Keywords: MACC1, colorectal cancer, prognosis, meta-analysis

Received: May 13, $2016 \quad$ Accepted: July 19, $2016 \quad$ Published: August 14, 2016

\section{ABSTRACT}

Metastasis-associated in colon cancer-1 (MACC1) has been reported to be overexpressed in diverse human malignancies, and the increasing amount of evidences suggest that its overexpression is associated with the development and progression of many human tumors. However, the prognostic and clinicopathological value of MACC1 in colorectal cancer remains inconclusive. Therefore, we conducted this metaanalysis to investigate the effect of MACC1 overexpression on clinicopathological features and survival outcomes in colorectal cancer. PubMed, CNKI, and Wanfang databases were searched for relevant articles published update to December 2015. Correlation of MACC1 expression level with overall survival (OS), disease-free survival (DFS), and clinicopathological features were analyzed. In this meta-analysis, fifteen studies with a total of 2,161 colorectal cancer patients were included. Our results showed that MACC1 overexpression was significantly associated with poorer OS and DFS. Moreover, MACC1 overexpression was significantly associated with gender, localization, TNM stage, $\mathbf{T}$ stage, and $\mathbf{N}$ stage. Together, our meta-analysis showed that MACC1 overexpression was significantly associated with poor survival rates, regional invasion and lymph-node metastasis. MACC1 expression level can serve as a novel prognostic factor in colorectal cancer patients.

\section{INTRODUCTION}

Colorectal cancer (CRC), which is the third most common cause of cancer and the third most common cause of cancer death after lung cancer, prostate cancer in men, and breast cancer in women, is a worldwide disease [1]. Every year, more than 1.2 million patients are diagnosed with colorectal cancer, from which more than 600,000 die [2]. Metastases arising from residual colorectal tumors is the major source of cancer-related deaths, approximately $90 \%$ of patient deaths. 5-year survival rates for patients with early stage, regional lymph node metastasis, and metastatic diseases are $\geq 90 \%, 65 \%$, and $\leq 10 \%$, respectively [3]. Therefore, early discovery of tumor occurrence and metastasis is critical to modify therapeutic strategies and improve patient prognosis. However, it is not determined to accurately predict the development of metastasis of CRC on the basis of the current clinicohistopathological classifications and molecular biomarkers. Therefore, the predictive biomarker for the early and accurate identification of high risk for metastasis in patients with CRC is ought to help to improve the clinical individual therapy.

Metastasis-associated in colon cancer-1 (MACC1) gene located at $7 \mathrm{p} 21.1$ was identified by a genome-wide search for a set of differently expressed genes in primary and metastatic colon cancer [4]. In the cultured cells, MACC1 was able to promote proliferation, migration, and dissemination, and to regulate gene transcription via the hepatocyte growth factor (HGF) /mesenchymal-epithelial transition factor signaling pathways [4]. Subsequent clinical investigations showed that MACC1 might be useful in the prognostic classification of colorectal cancer patients and was a promising new target for intervention in metastasis [4-8]. However, the relationship between MACC1 expression level and prognostic / clinicopathological outcomes in CRC patients remains controversial. Therefore, we conducted this meta-analysis. 
Table 1: Main characteristics of all studies included in the meta-analysis

\begin{tabular}{|c|c|c|c|c|c|c|c|c|c|c|c|}
\hline First author & Year & $\begin{array}{l}\text { Patient } \\
\text { source }\end{array}$ & $\begin{array}{l}\text { Number } \\
\text { of patient }\end{array}$ & Stage & Method & $\begin{array}{l}\text { MACC1 } \\
\text { expression } \\
(\%)\end{array}$ & $\begin{array}{l}\text { Median(range) } \\
\text { Follow- } \\
\text { up(month) }\end{array}$ & Outcome & $\begin{array}{l}\text { Multivariate/ } \\
\text { Univariate }\end{array}$ & HR(95\%CI) & $\begin{array}{l}\text { Scores } \\
\text { of } \\
\text { study }\end{array}$ \\
\hline Schmid $^{20}$ & 2015 & Germany & 60 & I-III & RT-PCR & 51.7 & NR & DFS & Univariate & $\begin{array}{l}1.71(0.41- \\
7.23)\end{array}$ & 6 \\
\hline Koelzer ${ }^{10}$ & 2015 & Greece & 187 & I-IV & $\mathrm{IHC}$ & 58.3 & NR & NR & NR & NR & 6 \\
\hline Katharina ${ }^{11}$ & 2015 & Germany & 99 & I-III & RT-PCR & 35.4 & 56.0 & DFS & Multivariate & $\begin{array}{l}6.09(2.50- \\
14.85)\end{array}$ & 8 \\
\hline $\mathrm{Xu}^{9}$ & 2015 & China & 96 & I-IV & $\mathrm{IHC}$ & 75 & $13(4-21)$ & NR & NR & NR & 6 \\
\hline $\mathrm{Ge}^{14}$ & 2015 & China & 96 & II-IV & IHC & 53.1 & 30.27 & DFS & Multivariate & $\begin{array}{l}2.11(1.32- \\
3.38)\end{array}$ & 7 \\
\hline Yamamoto $^{12}$ & 2014 & Japan & 174 & I-IV & RT-PCR & 82.2 & 49.2 & DFS & Multivariate & $\begin{array}{l}2.27(1.01- \\
9,71)\end{array}$ & 8 \\
\hline Zhen $^{13}$ & 2014 & China & 323 & I-IV & RT-PCR & 52.3 & NR & OS & Multivariate & $\begin{array}{l}1.410(0.737- \\
2.699)\end{array}$ & 7 \\
\hline $\operatorname{Ren}^{17}$ & 2013 & America & 93 & I-II & $\mathrm{IHC}$ & 73.1 & $20(2-62)$ & NR & NR & NR & 6 \\
\hline $\mathrm{Liu}^{15}$ & 2013 & China & 128 & I-IV & $\mathrm{IHC}$ & 65.0 & NR & NR & NR & NR & 6 \\
\hline Kang $^{16}$ & 2013 & China & 317 & I-IV & $\mathrm{IHC}$ & 61.8 & NR & OS & Univariate & $\begin{array}{l}2.67(1.57- \\
4.55)\end{array}$ & 6 \\
\hline Isella $^{8}$ & 2013 & Italy & 64 & NR & RT-PCR & 79.7 & 33 & DFS & Multivariate & $\begin{array}{l}\text { 7.274(1.658- } \\
31.91)\end{array}$ & 7 \\
\hline Zhang ${ }^{18}$ & 2012 & China & 90 & I-IV & IHC & 86.7 & 47.1 & OS & Univariate & $\begin{array}{l}0.46(0.06- \\
3.50)\end{array}$ & 8 \\
\hline Zhou $^{19}$ & 2012 & China & 80 & I-III & $\mathrm{IHC}$ & 41.3 & NR & NR & NR & NR & 6 \\
\hline Stein $^{5}$ & 2012 & Germany & 294 & I-IV & RT-PCR & 49 & 28 & OS & Univariate & $\begin{array}{l}4.89(1.90- \\
12.59)\end{array}$ & 7 \\
\hline Stein ${ }^{4}$ & 2009 & Germany & 60 & I-III & RT-PCR & 30 & 47.2 & DFS & Univariate & $\begin{array}{l}3.340(1.820- \\
6.130)\end{array}$ & 6 \\
\hline
\end{tabular}

\section{RESULTS}

\section{Search results and study characteristics}

A total of thirty-five articles were initially identified using the search criteria delineated above. As shown in Figure 1, twenty were excluded owing to irrelevance to the analysis or insufficient primary outcome. There were fifteen studies included in this meta-analysis [4, 5, 8-20].

The characteristics of the fifteen studies are summarized in Table 1. Of fifteen publications, eleven assessed the relationship between MACC1 expression and CRC clinicopathological features. Ten studies evaluated the association of MACC1 expression and prognosis in patients with CRC (four evaluated OS and six evaluated DFS). A total of 2,161 patients from China, Greece, Germany, America, Italy, and Japan were enrolled with sample number ranging from 60 to 323. To observe MACC1 status in CRC, immunohistochemistry (IHC) was used in eight studies and reverse transcription-polymerase chain reaction (RT-PCR) was used in seven. The median positive rate of MACC1 was $60.2 \%(30.0 \%-86.7 \%)$. Meanwhile, the follow-up times ranged from 20 to 56.2 months (Table 1). The study quality was assessed using the Newcastle-Ottawa quality assessment scale, generating scores ranging from 6 to 8 with a mean of 6.7.

\section{Quantitative synthesis}

\section{MACC1 expression and clinicopathological parameters}

To investigate the association between MACC1 expression and clinicopathological features, we conducted the meta-analysis. Accordingly, our results showed that increased MACC 1 expression was significantly correlated to gender $(\mathrm{OR}=0.804,95 \% \mathrm{CI}=0.654-0.988$, fixed-effect model), localization $(\mathrm{OR}=2.669,95 \% \mathrm{CI}=1.586-4.492$, fixed-effect model $)$, TNM stage $(\mathrm{OR}=1.976,95 \% \mathrm{CI}=$ 1.495-2.612, fixed-effect model), $\mathrm{T}$ stage $(\mathrm{OR}=2.002$, $95 \% \mathrm{CI}=1.548-2.589$, fixed-effect model), and $\mathrm{N}$ stage $(\mathrm{OR}=3.182,95 \% \mathrm{CI}=1.472-6.877$, random-effect model). On the contrary, MACC1 overexpression was not found to be associated with age $(\mathrm{OR}=1.200,95 \%$ $\mathrm{CI}=0.834-1.726$, random-effect model), tumor size (OR 
Table 2: Meta-analysis for the association of increased MACC1 expression and clinicopathological features of CRC patients

\begin{tabular}{|c|c|c|c|c|c|c|c|c|}
\hline \multirow{2}{*}{ Clinicopathological features } & \multirow{2}{*}{$\begin{array}{l}\text { No.of } \\
\text { studies }\end{array}$} & \multirow{2}{*}{$\begin{array}{l}\text { No.of } \\
\text { patients }\end{array}$} & \multirow{2}{*}{ Model } & \multirow{2}{*}{ OR(95\% CI) } & \multirow{2}{*}{$P$-Value } & \multicolumn{3}{|c|}{ Heterogeneity } \\
\hline & & & & & & $\overline{\chi^{2}}$ & $I^{2}(\%)$ & $P$-Value \\
\hline Age(younger $v s$. older) & 10 & 1500 & Random & $1.200(0.834,1.726)$ & 0.326 & 21.47 & 58.1 & 0.011 \\
\hline Size(smaller vs. bigger) & 7 & 994 & Random & $1.475(0.798,2.728)$ & 0.215 & 22.53 & 73.4 & 0.001 \\
\hline Gender(male vs. female) & 11 & 1682 & Fixed & $0.804(0.654,0.988)$ & 0.038 & 11.09 & 9.8 & 0.351 \\
\hline $\begin{array}{l}\text { Localization(Colon } \quad v s . \\
\text { Rectum) }\end{array}$ & 3 & 319 & Fixed & $2.669(1.586,4.492)$ & 0.000 & 2.71 & 26.1 & 0.258 \\
\hline Tumor grade(G1-2 vs. G3-4) & 3 & 382 & Fixed & $1.329(0.851,2.076)$ & 0.211 & 1.02 & 0.0 & 0.599 \\
\hline TNM stage(I-II vs. III-IV) & 6 & 891 & Fixed & $1.976(1.495,2.612)$ & 0.000 & 6.01 & 16.8 & 0.305 \\
\hline T stage(Tis-T2 vs. T3-T4) & 8 & 1306 & Fixed & $2.002(1.548,2.589)$ & 0.000 & 13.61 & 48.6 & 0.059 \\
\hline $\mathrm{N}$ stage(N0 vs. N1-N2) & 6 & 1034 & Random & $3.182(1.472,6.877)$ & 0.003 & 33.09 & 84.9 & 0.000 \\
\hline $\begin{array}{l}\text { Distant metastasis(M0 vs. } \\
\text { M1) }\end{array}$ & 6 & 1128 & Random & $1.925(0.761,4.870)$ & 0.167 & 17.44 & 71.3 & 0.004 \\
\hline
\end{tabular}

$=1.475,95 \% \mathrm{CI}=0.798-2.728$, random-effect model $)$, tumor grade $(\mathrm{OR}=1.329,95 \% \mathrm{CI}=0.851-2.076$, fixedeffect model $)$, or distant metastasis $(\mathrm{OR}=1.925,95 \%$
$\mathrm{CI}=0.761-4.870$, random-effect model). These results suggested that CRC with overexpressed MACC1 exhibited aggressive biological behaviors (Table 2).

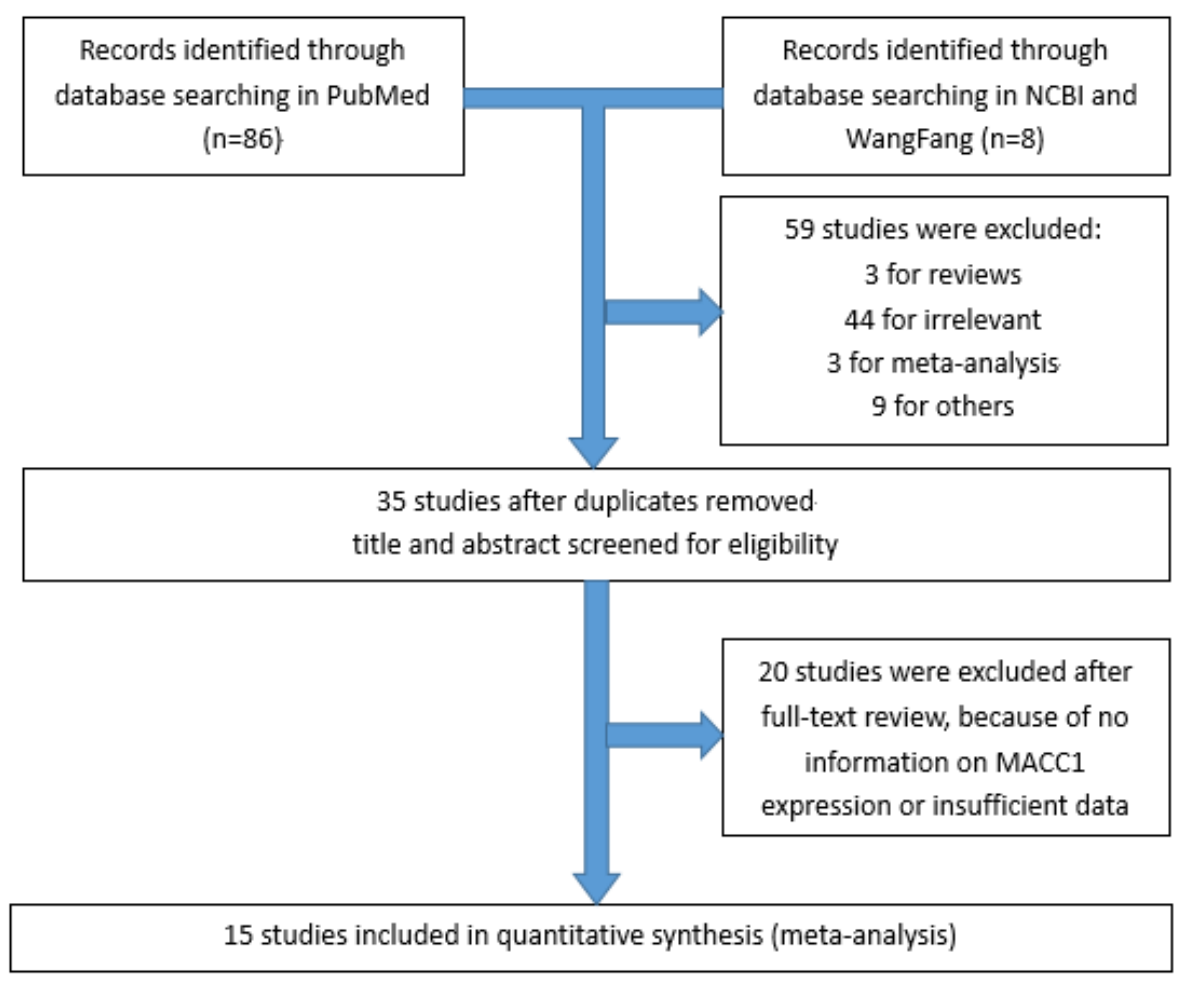

Figure 1: Flow chart of the selection of the studies in the meta-analysis. 
Table 3: Main meta-analysis results

\begin{tabular}{|c|c|c|c|c|c|c|c|}
\hline \multirow{2}{*}{ Analysis } & \multirow{2}{*}{ No.of studies } & \multirow{2}{*}{ No.of patients } & \multirow{2}{*}{ Model } & \multirow{2}{*}{ HR(95\% CI) } & \multirow{2}{*}{$P$-Value } & \multicolumn{2}{|c|}{ Heterogeneity } \\
\hline & & & & & & \begin{tabular}{|l|}
$\mathrm{I}^{2}$ \\
$\mathbf{( \% )}$ \\
\end{tabular} & $P$-Value \\
\hline OS & 4 & 1024 & Random & $2.16(1.12,4.18)$ & 0.022 & 59.0 & 0.062 \\
\hline \multicolumn{8}{|l|}{ Ethnicity } \\
\hline Asian & 3 & 730 & Random & $1.718(0.863,3.417)$ & 0.123 & 52.8 & 0.120 \\
\hline Non-Asian & 1 & 294 & - & $4.890(1.900,12.588)$ & 0.001 & - & - \\
\hline \multicolumn{8}{|l|}{ Method } \\
\hline IHC & 2 & 407 & Random & $1.474(0.289,7.524)$ & 0.641 & 62.8 & 0.101 \\
\hline RT-PCR & 2 & 617 & Random & $2.499(0.742,8.421)$ & 0.139 & 77.9 & 0.034 \\
\hline \multicolumn{8}{|l|}{ HR estimate } \\
\hline $\begin{array}{l}\text { Multivariate } \\
\text { analysis }\end{array}$ & 1 & 323 & - & $1.410(0.737,2.698)$ & 0.299 & - & - \\
\hline $\begin{array}{l}\text { Univariate } \\
\text { analysis }\end{array}$ & 3 & 701 & Random & $2.598(1.106,6.102)$ & 0.028 & 54.5 & 0.111 \\
\hline DFS & 6 & 553 & Fixed & $2.86(2.09,3.91)$ & 0.000 & 26.6 & 0.235 \\
\hline \multicolumn{8}{|l|}{ Ethnicity } \\
\hline Asian & 2 & 270 & Fixed & 2.133(1.382,3.292) & 0.001 & 0.0 & 0.907 \\
\hline Non-Asian & 4 & 283 & Fixed & $3.921(2.497,6.155)$ & 0.000 & 5.1 & 0.367 \\
\hline \multicolumn{8}{|l|}{ Method } \\
\hline IHC & 1 & 96 & - & $2.110(1.319,3.376)$ & 0.002 & - & - \\
\hline RT-PCR & 5 & 457 & Fixed & $3.638(2.392,5.531)$ & 0.000 & 0.0 & 0.415 \\
\hline \multicolumn{8}{|l|}{ HR estimate } \\
\hline $\begin{array}{l}\text { Multivariate } \\
\text { analysis }\end{array}$ & 4 & 433 & Random & $3.346(1.756,6.374)$ & 0.000 & 50.4 & 0.109 \\
\hline $\begin{array}{l}\text { Univariate } \\
\text { analysis }\end{array}$ & 2 & 120 & Fixed & $3.017(1.725,5.278)$ & 0.000 & 0.0 & 0.400 \\
\hline
\end{tabular}

OS, Overall survival; DFS, Disease-free survival

\section{MACC1 expression and OS in colorectal cancer}

Overall, four studies, including 1,024 patients, had a relationship between OS and MACC1 expression level. Heterogeneity among studies was statistically significant
$\left(P=0.062, \mathrm{I}^{2}=59.0 \%\right)$, so a random-effects model was used. The pooled HR for OS showed overexpression of MACC1 was significantly associated with reduced OS in CRC $(\mathrm{HR}=2.16,95 \% \mathrm{CI}=1.12-4.18, P=0.022$, Table 3 and Figure 2). We also performed subgroup analysis for 
ethnicity, method, and HR estimate. Subgroup analysis for ethnicity showed an insignificant association in Asian studies $(\mathrm{HR}=1.718,95 \% \mathrm{CI}=0.863-3.417, P=0.123)$. In the subgroup analysis for method of detection, the results suggested that the detection method, either RT-PCR or IHC, of MACC1 expression did not significantly influence outcomes (RT-PCR: $\mathrm{HR}=2.499,95 \% \mathrm{CI}=0.742-8.421$, $P=0.139$; IHC: $\mathrm{HR}=1.474,95 \% \mathrm{CI}=0.289-7.524, P=$ $0.641)$. For HR estimation, subgroup analysis suggested that the overall $\mathrm{HR}$ estimate for OS with univariate analysis was $2.598(95 \% \mathrm{CI}=1.106-6.102, P=0.028)$ (Table 3).

\section{MACC1 expression and DFS in colorectal cancer}

Six studies comprising a total 553 patients provided results regarding to DFS. There was no significant heterogeneity $(P=0.235$, I $2=26.6 \%)$ among them, so a fixed-effect model was used to calculate the pooled HR and $95 \% \mathrm{CI}$. Our results showed that increased MACC1 expression was significantly associated with poorer DFS $(\mathrm{HR}=2.86,95 \% \mathrm{CI}=2.09-3.91, P=0.000)$, indicating that increased MACC1 expression was an indicator of disease recurrence in CRC patients (Table 3 and Figure 3). Meanwhile, a tight association was also observed between MACC1 expression and CRC patient prognosis across various geographic regions; the pooled HR was 2.133
$(95 \% \mathrm{CI}=1.382-3.292, P=0.001)$ from Asia, and HR was characterized as $3.921(95 \% \mathrm{CI}=2.497-6.155, P=0.000)$ from non-Asian countries. Subgroup analysis by method and HR estimation suggested a significant association in RT-PCR $(\mathrm{HR}=3.638,95 \% \mathrm{CI}=2.392-5.531, P=0.000)$, multivariate analysis $(\mathrm{HR}=3.346,95 \% \mathrm{CI}=1.756-6.374$, $P=0.000)$ and Univariate analysis $(\mathrm{HR}=3.017,95 \% \mathrm{CI}$ $=1.725-5.278, P=0.000)$ (Table 3 ).

\section{Publication bias}

In this meta-analysis, both Begg's and Egger's tests were performed to assess if any publication bias existed in the published literature. No publication bias was observed among studies with OS $(P=1.000,0.679)$ and DFS $(P=$ $0.707,0.433)$. The Begg's plots for the effect of MACC1 expression level on prognosis were shown in Figures 4A and $4 \mathrm{~B}$.

\section{DISCUSSION}

Identification of a novel predictive and prognostic marker to guide clinical therapy for patients with CRC is currently of special interest. Many molecular markers, such as TP53 [21], KRAS, and BRAF [22], have been

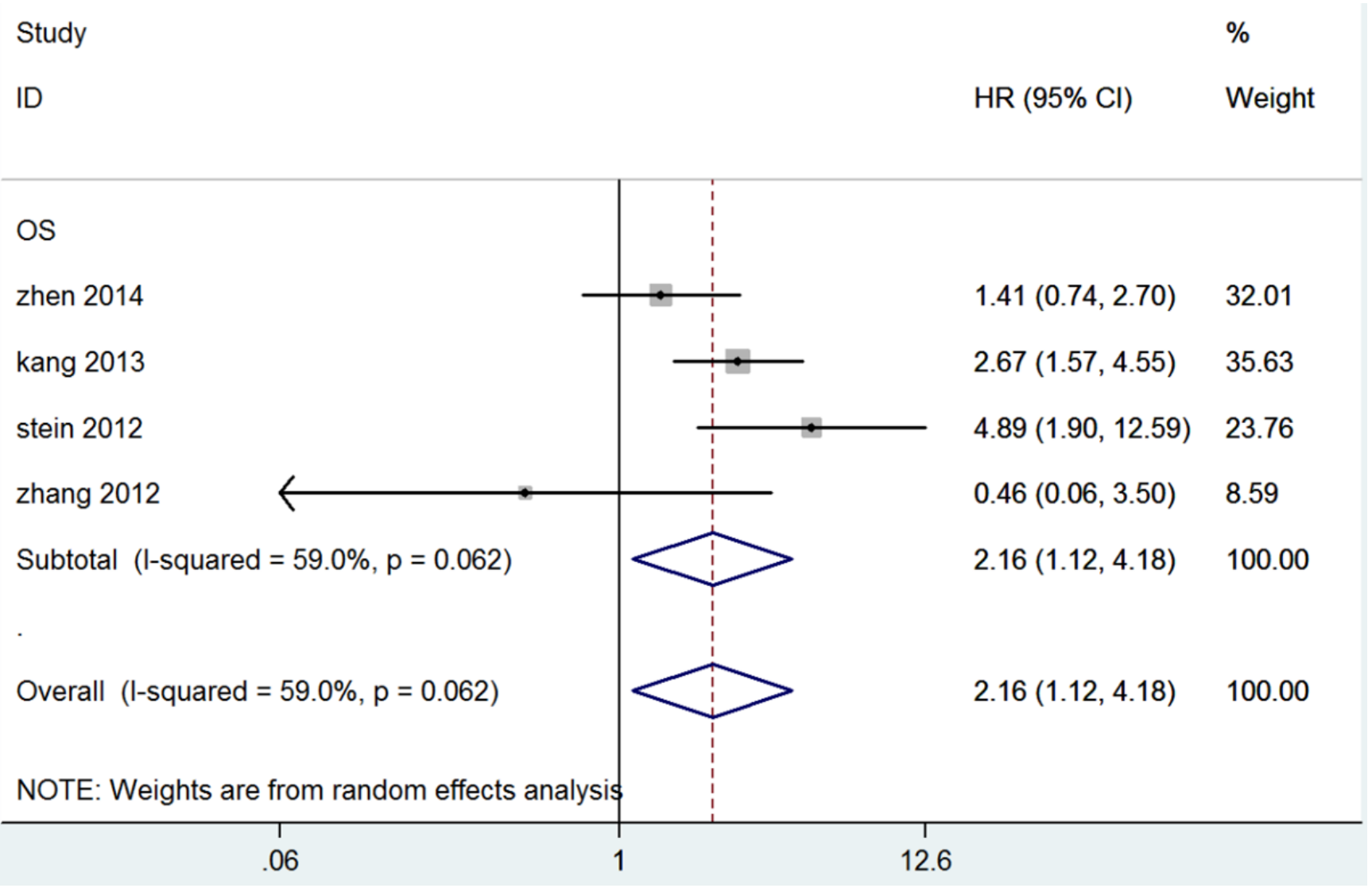

Figure 2: Forest plot of hazard ratio for the association of MACC1 overexpression and overall survival. 
investigated over the past few years. Notwithstandingly, they are not routinely used in clinical practice due to their accuracy and stability to predict the prognosis of CRC. In recent years, some studies have shown the potential clinical value of MACC1 to serve as a prognostic indicator and a potential novel target for treatment in patients with CRC.

The first study investigating the relationship between MACC1 and survival patients with cancers was reported by Stein et al. Their results suggested that MACC1 expression level acted as an independent prognostic indicator of tumor metastasis and diseasefree survival (DFS) [4]. Recently, a number of studies have been carried out to investigate the correlation of MACC1 expression to the survival and prognosis of CRC patients, but results have been inconsistent. Meta-analysis have been performed to resolve controversial results for identification of prognostic indicators in patients with malignant diseases, and more recently, this approach has been applied widely and successfully. Therefore, we conducted a meta-analysis of the evidence obtained from all published MACC1/CRC studies in order to provide a quantitative reassessment of this association.
The present study was the meta-analysis of published data regarding to the relationship between MACC1 and disease prognosis in patients with CRC. We observed a relationship between MACC1 overexpression, poorer overall survival, and disease-free survival, and among subgroups defined by study region. Furthermore, we observed an association between MACC1 overexpression and several clinicopathological parameters (patient age, tumor size, patient gender, cancer localization, tumor grade, TNM stage, nodal status, tumor depth, and distant metastases). This meta-analysis engenders several important implications. First of all, we observed a positive relationship between MACC1 overexpression and several clinicopathological parameters. Females with colon cancer displayed a poorer prognosis as compared to males with rectal cancer. In addition, the pooled results of TNM stage, nodal status, and tumor depth suggested that increased MACC 1 expression promoted regional invasion and lymph-node metastasis, thus leading to poorer CRC prognosis. However, we can also see from the results that increased MACC1 expression was not correlated with distant metastasis. This lead us to conclude that MACC1 overexpression might be associated with CRC

Study

$\%$

ID

HR $(95 \% \mathrm{Cl}) \quad$ Weight

\section{DFS}

schmid 2015

katharina 2015

ge 2015

yamamoto 2014

isella 2013

stein 2009

Subtotal $(\mathrm{I}$-squared $=26.6 \%, p=0.235$ )

Heterogeneity between groups: $p=$.

Overall (I-squared $=26.6 \%, p=0.235$ )

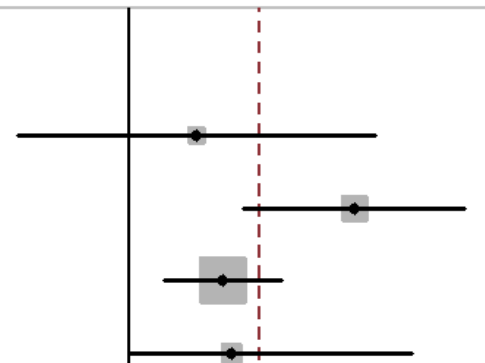

$1.71(0.41,7.23) \quad 4.75$

$6.09(2.50,14.85) \quad 12.33$

$2.11(1.32,3.38) \quad 44.27$

$2.27(1.01,9.71) \quad 7.64$

$7.27(1.66,31.91) \quad 4.47$

$3.34(1.82,6.13) \quad 26.54$

$2.86(2.09,3.91) \quad 100.00$

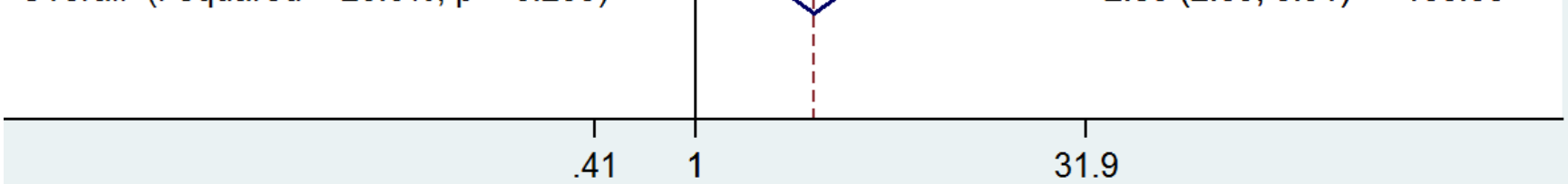

Figure 3: Forest plot of hazard ratio for the association of MACC1 overexpression and disease-free survival. 


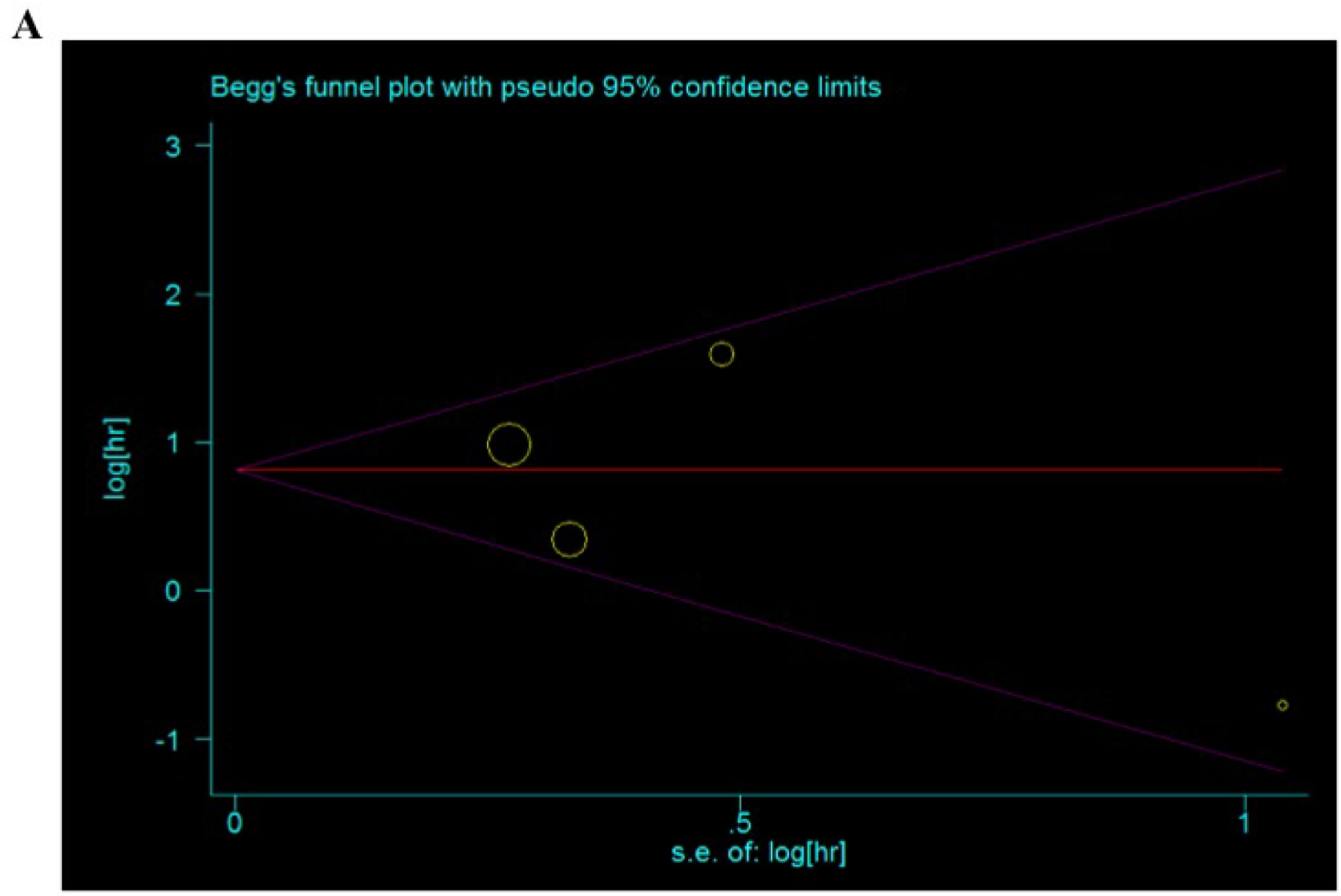

B

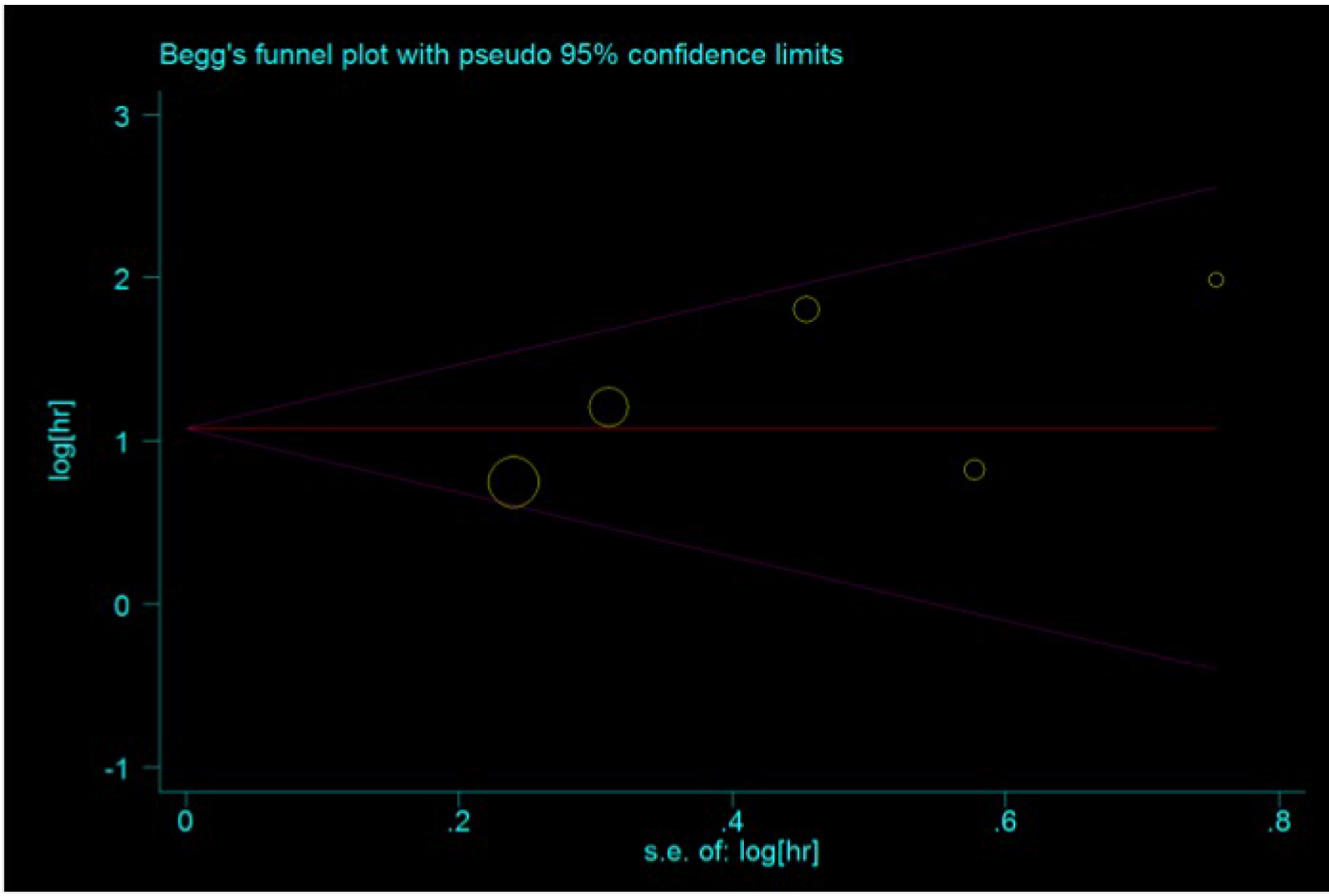

Figure 4: Funnel plots of publication bias in this meta-analysis. A. Overall survival; B. Disease-free survival. 
progression, but this has to be proved by research using sufficiently large sample sizes. Pooled statistical data, however, did show that overexpression of MACC1 was associated with worse survival outcomes, including OS and DFS. These results indicated that MACC1 might serve as a new parameter for predicting outcomes and a potential novel target for treatment in CRC patients. Finally, when extended to subgroup analysis of analysis method of MACC1, The same conclusion is found between RTPCR and IHC. Similarly, from the point of ethnicity and HR estimate, we also have come to the same conclusion, although this may result from too-small sample sizes, causing a potential type I error. Therefore, it is necessary to perform better-designed studies using huge sample to confirm or to refute our findings.

In our meta-analysis, we found significant heterogeneity across the included studies. The $\mathrm{I}^{2}$ values for OS and DFS were $59.0 \%$ and $26.6 \%$, respectively. Although we used random-effect models to pool the OS data, the models did not identify the source of heterogeneity. In addition, a random-effect model can reduce the effect of large-sample studies of good quality. Although the exact sources of heterogeneity were not well-clarified, there were several possible reasons for this heterogeneity, such as the detection method for measuring MACC1 expression levels, the small number of included studies, the differences in TNM stage, and the statistical approach for extrapolating HRs. However, since we found no publication bias, statistical results seemed to be robust and convincing. The data on MACC1 expression and CRC prognosis is promising, but strongly supported the further clinical study to uncover the potential and value of $\mathrm{MACC} 1$ to function as a prognoticator.

In conclusion, we found that MACC1 expression indicated poor survival outcomes and regional invasion and lymph-node metastasis. Therefore, we believe that MACC1 can serve as a prognostic indicator and a potential novel target for treatment in CRC patients. Larger and more well-designed studies are required to clarify the prognostic significance of MACC1 expression in CRC patients.

\section{MATERIALS AND METHODS}

This meta-analysis was conducted in accordance with the Preferred Reporting Items for Systematic Reviews and Meta-Analyses (PRISMA) guidelines.

\section{Identification and selection of studies}

Studies were identified by searching PubMed, CNKI, and WanFang databases covering all papers published update to December 2015. The following search strategy was used: "colon cancer or colon carcinoma or rectum cancer or rectum carcinoma or colorectal cancer or colorectal carcinoma" and "MACC1 or Metastasisassociated in colon cancer-1". No language restrictions were applied. All eligible studies were retrieved, and their references were cross-searched to triage additional suitable studies. Once publications were found with overlapping data published by the same investigator, only the most complete report was included. Disagreements were resolved by iteration, discussion, and consensus between the two authors.

Studies were included if they fulfilled the following criteria: (a) reporting explicit methods for the detection of MACC1 expression in CRC; (b) the endpoints were to evaluate the relationship of MACC1 expression in CRC patients with OS, DFS, and a series of clinicopathological parameters; and (c) provided a hazard ratio (HR) or odds ratio (OR) with the corresponding confidence interval (CI) or sufficient data to calculate them. Articles were excluded from the analysis following criteria: (a) letters, case reports, reviews, and conference abstracts without original data; (b) duplicates of previous publications; (c) articles without key information such as Kaplan-Meier curves, hazard ratios (HRs) with the 95\% confidence intervals (CIs), or clinicopathological features.

\section{Data extraction}

Two independent reviewers extracted the details of included studies with a standardized form. The following information was recorded: first author's surname, year of publication, number of patient, patient source, tumor stage, MACC1 assessment method, MACC1 expression, followup time, prognostic outcomes, analytical method, and HR with its $95 \%$ CI. If the above-mentioned data was not reported, items should be treated as "NR (not reported)".

\section{Methodological quality of the studies}

The Newcastle-Ottawa Scale was used to assess the quality of each study [23]. The NOS criteria is scored based on three aspects: (1) subject selection, (2) comparability of subject, (3) clinical outcome. NOS scores range from 0 to 9 , and a score $\geq 6$ indicates a high quality. Two investigators independently assessed the quality of the 9 included studies, and the discrepancies were solved by consensus.

\section{Statistical methods}

Included studies were divided into three groups: OS, DFS, and clinicopathological parameters. MACC1 was considered as 'high' or 'low' expression according to the cut-off values provided by the authors in each publication, because of variation on the definition for the 'high' or 'low' expression of MACC1 among studies. 
Hazard ratios (HRs) and 95\% CIs were combined to measure the effective value. For these HRs that were given explicitly in the published studies, we used crude ones. If not, we calculated the values from the KaplanMeier survival curve or the available data using methods reported by Parmar et al [24]. Data from the Kaplan-Meier survival curves were read using Engauge Digitizer version 4.1. A combined HR/OR $>1$ indicated a poor outcome for MACC1 overexpression, while $\mathrm{HR} / \mathrm{OR}<1$ indicated a favorable outcome for MACC1 overexpression. Heterogeneity among the studies was determined by chisquare test and Q test. If heterogeneity was significant $(P$ $<0.1$ or $\mathrm{I}^{2}>50 \%$ ), the DerSimonian and Laird randomeffects model were used [25]. Otherwise, a fixed-effects model of Mantel-Haenszel was applied in the absence of between-study heterogeneity [26]. Both Egger's and Begg's tests were used to examine publication bias $[27,28]$. All $P$ values were two-sided, and $P<0.05$ was considered as statistically significant. Statistical calculations were performed using STATA 12.0.

\section{Abbreviations}

MACC $1=$ metastasis-associated in colon cancer-1, $\mathrm{CRC}=$ colorectal cancer, $\mathrm{HR}=$ hazard ratios, $\mathrm{OR}=$ odds ratio, $\mathrm{CI}=$ confidence interval, $\mathrm{OS}=$ overall survival, $\mathrm{DFS}$ $=$ disease-free survival, $\mathrm{IHC}=$ immunohistochemistry, RT$\mathrm{PCR}=$ reverse transcription-polymerase chain reaction.

\section{ACKNOWLEDGMENTS}

Thanks for the language editing by Editage.

\section{CONFLICTS OF INTEREST}

No.

\section{GRANT SUPPORT}

This study was supported by National Natural Science Foundation, China (No. 81471670, 81301847); China Postdoctoral Science Foundation (No. 2015T81037); Science and Technology Plan of Innovation Project, Shaanxi province, China (No. 2015KTCL03-06) and the Fundamental Research Funds for the Central Universities, China (No. 2014qngz-04).

\section{Author Contributions}

Zhijun Dai and Cong Dai contributed to the design of the study and manuscript writing. Yang Zhao, Cong Dai and Huafeng Kang contributed to the data extraction and analysis process of the study. Shuai Lin, Pengtao Yang, Xinghan Liu, and Kang Liu carried out statistical analysis.
Yang Zhao, Xu Peng, Yi Zheng, Shanli Li, and Cong Dai prepared all figures and tables. Zhijun Dai, Yang Zhao, and Cong Dai contributed to revision of the manuscript. All authors reviewed the manuscript.

\section{REFERENCES}

1. Siegel RL, Miller KD and Jemal A. Cancer statistics, 2015. CA Cancer J Clin. 2015; 65:5-29.

2. Brenner H, Kloor M and Pox CP. Colorectal cancer. Lancet. 2014; 383:1490-1502.

3. Stein U and Schlag PM. Clinical, biological, and molecular aspects of metastasis in colorectal cancer. Recent Results Cancer Res. 2007; 176:61-80.

4. Stein U, Walther W, Arlt F, Schwabe H, Smith J, Fichtner I, Birchmeier W and Schlag PM. MACC1, a newly identified key regulator of HGF-MET signaling, predicts colon cancer metastasis. Nat Med. 2009; 15:59-67.

5. Stein U, Burock S, Herrmann P, Wendler I, Niederstrasser M, Wernecke KD and Schlag PM. Circulating MACC1 transcripts in colorectal cancer patient plasma predict metastasis and prognosis. Plos One. 2012; 7:e49249.

6. Kawamura M, Saigusa S, Toiyama Y, Tanaka K, Okugawa Y, Hiro J, Uchida K, Mohri Y, Inoue Y and Kusunoki M. Correlation of MACC1 and MET expression in rectal cancer after neoadjuvant chemoradiotherapy. Anticancer Res. 2012; 32:1527-1531.

7. Nitsche U, Rosenberg R, Balmert A, Schuster T, SlottaHuspenina J, Herrmann P, Bader FG, Friess H, Schlag PM, Stein U and Janssen KP. Integrative marker analysis allows risk assessment for metastasis in stage II colon cancer. Ann Surg. 2012; 256:763-771; discussion 771.

8. Isella C, Mellano A, Galimi F, Petti C, Capussotti L, De Simone M, Bertotti A, Medico E and Muratore A. MACC1 mRNA levels predict cancer recurrence after resection of colorectal cancer liver metastases. Ann Surg. 2013; 257:1089-1095.

9. Xu L, Xuan Z, Lin G, Qiu H, Xiao Y and Wu B. Expression of MACC1 protein in colorectal cancer and its relationship with clinicopathological characteristics. Chin J Gastrointest Surg. 2015; 18:1032-1035.

10. Koelzer VH, Herrmann P, Zlobec I, Karamitopoulou E, Lugli A and Stein U. Heterogeneity analysis of Metastasis Associated in Colon Cancer 1 (MACC1) for survival prognosis of colorectal cancer patients: a retrospective cohort study. BMC Cancer. 2015; 15:160.

11. Katharina I, Kemmner W, Osterland M, Burock S, Koch G, Herrmann P, Schlag PM and Stein U. High MACC1 expression in combination with mutated KRAS G13 indicates poor survival of colorectal cancer patients. Mol Cancer. 2015; 14:38.

12. Yamamoto H, Miyoshi N, Mimori K, Hitora T, Tokuoka M, Fujino S, Ellis H, Ishii H, Noura S, Ohue M, Yano M, Doki Y and Mori M. MACC1 expression levels as a novel 
prognostic marker for colorectal cancer. Oncol Lett. 2014; 8:2305-2309.

13. Zhen T, Dai S, Li H, Yang Y, Kang L, Shi H, Zhang F, Yang D, Cai S, He Y, Liang Y and Han A. MACC1 promotes carcinogenesis of colorectal cancer via betacatenin signaling pathway. Oncotarget. 2014; 5:3756-3769. doi: 10.18632/oncotarget.1993.

14. Ge Y, Meng X, Zhou Y, Zhang J and Ding Y. Positive MACC1 expression correlates with invasive behaviors and postoperative liver metastasis in colon cancer. Int J Clin Exp Med. 2015; 8:1094-1100.

15. Liu X, Liu JC, Yu D, Cai Q, Zhou HC, Peng GX and He F. Expression and Clinicopathological Significance of MACC1 and nm23H1 in Colorectal Cancer. Mod Diagn Treat. 2013; 24:4054-4060.

16. Kang AD, Fang YP, Deng NN, Gao HU and Guo B. Expression and Clinicopathological Significance of MACC1 in Colon Cancer. Progress in Modern Biomedicine. 2013; 13:5087-5090.

17. Ren B, Zakharov V, Yang Q, McMahon L, Yu J and Cao W. MACC1 is related to colorectal cancer initiation and early-stage invasive growth. Am J Clin Pathol. 2013; 140:701-707.

18. Zhang XH and Jiang WZ. Expression of MACC1 Protein in Rectal Adenocarcinoma and Its Clinical Significance. Medical Innovation of China. 2012; 9:24-26.

19. Zhou ZH, Chen SZ and Liao HQ. The Expression and Clinical Significance of MACC1 Gene in Colon Cancer. The Practical Journal of Cancer. 2012; 27:30-31.

20. Schmid F, Wang Q, Huska MR, Andrade-Navarro MA, Lemm M, Fichtner I, Dahlmann M, Kobelt D, Walther W, Smith J, Schlag PM and Stein U. SPON2, a newly identified target gene of MACC1, drives colorectal cancer metastasis in mice and is prognostic for colorectal cancer patient survival. Oncogene. 2015.
21. Westra JL, Schaapveld M, Hollema H, de Boer JP, Kraak MM, de Jong D, ter Elst A, Mulder NH, Buys CH, Hofstra RM and Plukker JT. Determination of TP53 mutation is more relevant than microsatellite instability status for the prediction of disease-free survival in adjuvant-treated stage III colon cancer patients. J Clin Oncol. 2005; 23:5635-5643.

22. Roth AD, Tejpar S, Delorenzi M, Yan P, Fiocca R, Klingbiel D, Dietrich D, Biesmans B, Bodoky G, Barone C, Aranda E, Nordlinger B, Cisar L, Labianca R, Cunningham D, Van Cutsem E, et al. Prognostic role of KRAS and BRAF in stage II and III resected colon cancer: results of the translational study on the PETACC-3, EORTC 40993, SAKK 60-00 trial. J Clin Oncol. 2010; 28:466-474.

23. Stang A. Critical evaluation of the Newcastle-Ottawa scale for the assessment of the quality of nonrandomized studies in meta-analyses. Eur J Epidemiol. 2010; 25:603-605.

24. Parmar MK, Torri V and Stewart L. Extracting summary statistics to perform meta-analyses of the published literature for survival endpoints. Stat Med. 1998; 17:28152834.

25. DerSimonian R and Laird N. Meta-analysis in clinical trials. Control Clin Trials. 1986; 7:177-188.

26. Mantel $\mathrm{N}$ and Haenszel W. Statistical aspects of the analysis of data from retrospective studies of disease. J Natl Cancer Inst. 1959; 22:719-748.

27. Egger M, Davey Smith G, Schneider M and Minder C. Bias in meta-analysis detected by a simple, graphical test. Bmj. 1997; 315:629-634.

28. Begg CB and Mazumdar M. Operating characteristics of a rank correlation test for publication bias. Biometrics. 1994; 50:1088-1101. 